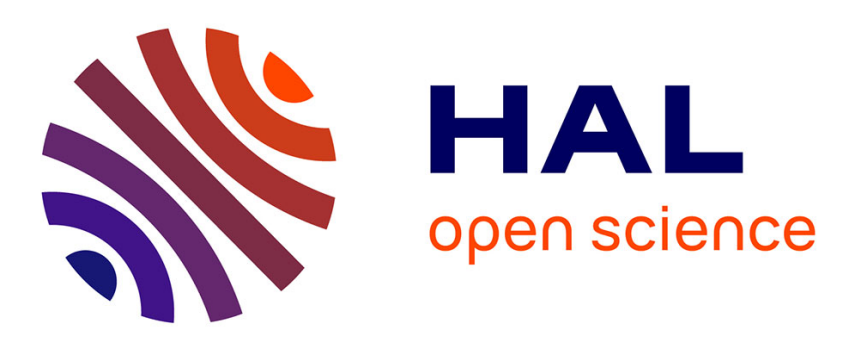

\title{
Navigation Strategy In-flight Retrieving and Transportation Operations for a Rotorcraft MAV
}

Juan Escareño, Jesus Castillo, Wafik Abassi, Gerardo Flores, Karla Camarillo

\section{To cite this version:}

Juan Escareño, Jesus Castillo, Wafik Abassi, Gerardo Flores, Karla Camarillo. Navigation Strategy In-flight Retrieving and Transportation Operations for a Rotorcraft MAV. 4th Workshop on Research, Education and Development of Unmanned Aerial Systems (RED-UAS 2017), Oct 2017, Linköping, Sweden. (elec. proc.). hal-01675011

\section{HAL Id: hal-01675011 https://hal.science/hal-01675011}

Submitted on 3 Jan 2018

HAL is a multi-disciplinary open access archive for the deposit and dissemination of scientific research documents, whether they are published or not. The documents may come from teaching and research institutions in France or abroad, or from public or private research centers.
L'archive ouverte pluridisciplinaire HAL, est destinée au dépôt et à la diffusion de documents scientifiques de niveau recherche, publiés ou non, émanant des établissements d'enseignement et de recherche français ou étrangers, des laboratoires publics ou privés. 


\title{
Navigation Strategy In-flight Retrieving and Transportation Operations for a Rotorcraft MAV
}

\author{
J. Escareno, J. Castillo, W. Abassi, G. Flores and K. Camarillo
}

\begin{abstract}
The paper addresses a robust navigation strategy of a rotorcraft class having a simple robotic arm to perform rapid in-flight retrieving operations in presence of wind gusts and aerodynamic effects. The target-acquiring trajectory planner is based on a cosinusoidal dynamic pattern. The mathematical model is extended to account not only the dynamics of the robotic arm but also the aerodynamics. The navigation control scheme is based on a soft integral slidingmode control (ISC) to stabilize both inner- and outer-loop dynamics regarding the rejection of not only the dynamic couplings but also aerodynamic disturbances. Detailed simulations including realistic aerodynamic effects results show the validity of the proposed navigation strategy while tracking the objectto-retrieve trajectory.
\end{abstract}

\section{INTRODUCTION}

In the recent years, Unmanned Aerial Vehicles (UAVs), specially Micro Aerial Vehicles (MAVs), have been used for different tasks, included but not limited to industrial or scientific ones. Due to its mechanical simplicity and operational versatility, they encourage the rise of new applications. The technological and scientific challenges associated to these emergent generation of aerial robots are enormous.

A recent and pertinent application is aerial interactivity of MAVs with the environment, such interactivity must consider the effects of the wind and the disturbances generated by the payload. Some examples are in-contact structure inspection, aerial manipulation and transportation outdoors, where, in both cases, the wind becomes the main drawback during an aerial manipulation/grasping operation altering the behavior, performance and stability of the aerial robot.

Since most of UAV-applications evolve outdoors, the aerial robot is subject to external disturbances due to adverse atmospheric conditions that deteriorate the performance and navigation capability of the vehicle. These adverse effects (forces and torques) triggered by aerodynamic phenomena, such as drag, become critical as the MAV increases its velocity. For this reason, more efficient task-based patterns and robust control algorithms are required to achieve the motion control objective.

\section{A. Related Work}

Previous research works related to the motion control of MAVs operating in adversary wind conditions have particularly focused on fixed-wing configurations. In these attempts

J. Escareno and W. Abassi is with the Polytechnic Institute of Advanced Sciences, 63 Blvd. de Brandebourg, 94200 Ivry-sur-Seine, France, escareno, wafik.abassieipsa.fr.J. Escareno is also with the laboratory IBISC of Universite d'Evry, France.

J. Castillo and K. Camarillo are with the Instituto Tecnologico de Celaya, Av. Tecnológico, 38010 Celaya, Mexico, jesus. castillo, karla.camarillo@itcelaya.edu.mx. a constant wind speed up to $50 \%$ of MAV airspeed is assumed as a flight condition.

[1] experimentally implemented a path-following controller in which a constant-velocity MAV under moderate wind conditions (20\% to $50 \%$ of MAV airspeed) is used to prove the effectiveness of the controller. [2] introduces path planning and control algorithms meant to survey multiple waypoints while heading is considered to evolve at a constrained rate. The path planning task is considered to be an optimization problem where the constant wind component is assumed known, while the sliding surface controller is used to deal with small time varying wind components. A linear quadratic regulator for optimal guidance of a fixed-wing aerial vehicle is exposed in [3]. The controller, validated by simulations, aims to follow straight-line and circular trajectories under different wind conditions. [4] presents a nonlinear controller to make a MAV follow a straight-line reference successfully. Various constant lateral wind components (crosswind) up to $40 \%$ of the MAV speed are considered in this work.

Various are the efforts addressing the flight control of quadrotor MAVs under wind disturbances, in which the problem is approached at a dynamic level, i. e. including the wind forces in the overall dynamics as disturbances, either rotational or translational.

[5] focuses on robust position control of a quad-rotor by modeling the wind influence using the Dryden Wind Gust Model and including it in the dynamics of the vehicle aiming, by this way, at the estimation of the in-flight wind velocities. Moreover, [6] has dealt with the motion control problem implementing a hybrid backstepping controller that considers the desired accelerations obtained by the FrenetSerret Theory (Backstepping-FST). Simulations have shown that such control design provides a robust flight when the MAV faces wind disturbances $(0.8$ and $0.5 \mathrm{~m} / \mathrm{s})$ at moderate rotorcraft velocities $(2 \mathrm{~m} / \mathrm{s})$ during a target-tracking operation. On the other hand, [7] introduces a robust position control for the quad-rotor. The robust behavior is achieved via a disturbance observer that includes not only wind gust but also the nonlinear terms of the vehicle completely in a total disturbance force, which permits to obtain the linear dynamics and to treat them through standard PID controllers. In [8], a two-level controller as well as an observer to estimate the wind disturbance in the translational dynamics are proposed.

Rotorcraft MAVs used for acquiring and transporting cargo is a popular topic addressed in the last year due to its potential impact for a wide range of industrial applications (e.g. parcel delivering industry). In [9], a quad-rotors fleet transport a 
cargo through cables. The generation of trajectories where the quad-rotor provides swing-free load motion has attracted the interest of diverse authors. An alternative configuration equipped with a hook meant to retrieve/deliver cargo using a vision-based strategy is presented in [10]. [11] presents the modeling and control of a mini helicopter equipped with a compliant gripper capable of robust grasping and transporting. In [12], the authors present a classical quad-rotor equipped with a monocular camera. The proposed control strategy enables performing aggressive grasping maneuvers via an Image Based Visual Servoing (IBVS). In [13], a classical quad-rotor featuring a home-customized 1 degree of freedom (DOF) gripper performs an aerial grasping based on IR cameras.

\section{B. Paper contributions}

This paper addresses the trajectory pattern generation problem of a rotorcraft using a 1DOF manipulator robot for aerial payload picking-and-transporting considering aerodynamic adverse effects to this multi-body aerial configuration. Due to its geometry, the vehicle is exposed to significant aerodynamic and coupling disturbances, which must be considered in the dynamic equation, especially for aerial robots with such specifications. In the aforementioned works addressing wind-tolerant navigation, the aerodynamic disturbances are only considered at the translational level (outer-loop), meanwhile, in this paper, also those affecting the rotational dynamics (inner-loop) are considered. For this reason, we propose a trajectory planner based on the target's location and whose speed pattern is adapted to diminish the aerodynamic disturbances. The motion path, which includes position and velocity, is partially inspired on avian-like retrieving (e.g. the bird of prey features a cosinusoidallike path), where birds increase the angle of attack (AoA) at the target retrieval neighborhood damping their velocity due to drag. However, in the case of study here exposed, the rotorcraft keeps small AoAs while holding horizontal velocity at pick-up point.

An aerodynamic detailed study is provided regarding the most appropriate drone-gripper configuration to diminish the effects of the aerodynamic phenomena over the rotorcraft and the pendulum-like gripper at different velocities which allows to define an appropriate operational regime. In this regard, the Dryden model is used to recreate a realistic simulation environment [5].

\section{Outline}

This work is organized as follows: the problem statement, describing the main goals as well as the dynamic and aerodynamic modeling, is presented in Section II Section III presents the control scheme to mitigate the wind disturbance and thus accomplishing the tracking control objective. Numerical simulations considering a realistic scenario are detailed in Section IV Lastly, the conclusions and the perspectives are given in Section $\mathrm{V}$.

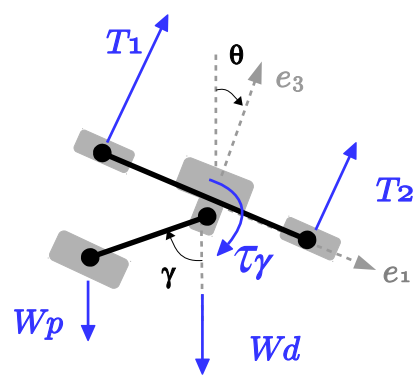

(a)

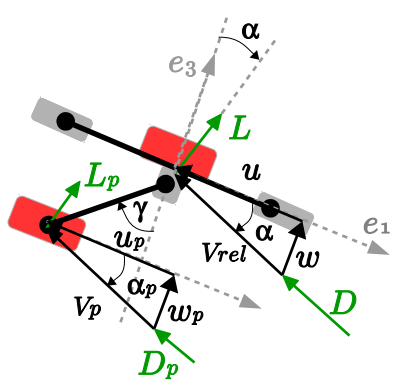

(b)
Fig. 1. (a) Forces exerted (b) aerodynamic situation

\section{Problem Formulation}

Let us consider the problem where a multi-rotor aerial robot, equipped with a robotic manipulator which is illustrated in Fig. 1 (a detailed description is presented in subsection II-A), is meant to perform in-flight pick-andplace operations while respecting specific speed patterns. For a given reference path $\mathcal{P}(t)$, it is required that the vehicle reaches a time-varying spatial point according to a timing law, where the two dimensional reference trajectory is assumed two-times differentiable and bounded.

Performing in-flight operations imply additional forces and torques exerted on the rotorcraft resulting for dynamic couplings, additionally, the presence of wind gust provokes erroneous flight trajectories shifting away the vehicle from the commanded path. For this reason, such unknown disturbances must be included in the control design, either via estimation and/or robust techniques. The flight profile of a rotorcraft is not constrained to minimum-positive velocity, as is the case of fixed-wing MAVs (stall angle), in fact it might generate negative velocities. This property makes this air platform ideal for tracking purposes under two-dimensional wind conditions.

\section{A. Mathematical Modeling}

Consider a class of multi-rotor robot, of mass $M \in \mathbb{R}$ and moment of inertia $I_{r} \in \mathbb{R}$, having an actuated 1DOF robotic arm attached to the main airframe featuring a length $\ell \in \mathbb{R}$ and capable of grasping a pendulum-like payload of mass $m \in \mathbb{R}$. For the actual study and for the sake of clarity, the aerial robot is consider to evolve within the longitudinal plane.

At this point, it is convenient to consider some assumptions to define the dynamic scenario where:

1) The structural values $M, I_{r}$ and $\ell$ are known;

2) The maximal translational velocity is restricted to $7 \mathrm{~m} / \mathrm{s}$

3) The payload to be acquired $(m)$ is assumed unknown but limited to $50 \%$ of vehicle's mass, i.e. $|m| \leq \frac{M}{2}$;

4) The mass of the arm is assumed small, and thus neglected. 


\section{B. Aerodynamics}

The current paper focuses on the vehicle operation in the longitudinal plane while it performs high-speed payload acquiring-and-deploying maneuvers. For this reason a detailed treatment of the vehicle's aerodynamics is given. Since the dynamic behavior is affected by aerodynamics for highspeed operational regimes, it is thus essential to consider aerodynamic forces properly. They are mainly produced by the vehicles motion, with respect to still air, or due to wind gusts impinging on a stationary rotorcraft or a combination of these. The aerodynamic modeling process is illustrated by Fig. 2 .

For the aerodynamic analysis, the following assumptions are made:

1) Propeller normal forces are negligible;

2) The vehicle's structure is fully submerged within the wind gusts streams;

3) The payload's geometry is considered spherical;

4) The horizontal velocity is much bigger than vertical, i.e. $\dot{x}>>\dot{z}$.

A real-world flight envelope contains not only the velocity vector from vehicle's motion, either in the body $\left(\mathbf{V}^{\mathcal{B}}=\right.$ $\left.(u, w)^{T} \in \mathbb{R}^{2}\right)$ or inertial $\left(\mathbf{V}^{\mathcal{I}}=(\dot{x}, \dot{z})^{T} \in \mathbb{R}^{2}\right)$, but also it is exposed to windy conditions. For this reason, in order to continue with the aerodynamic analysis it is important to detail, for a close-to-reality simulation, the generation of the wind field. The wind vector $\mathbf{V}_{w}^{\mathcal{I}}=\left(v_{w_{x}}, v_{w_{z}}\right)^{T} \in \mathbb{R}^{2}$, is obtained from the Dryden spectral model [5], it is composed by the static and the turbulent wind components. Thus, the vertical and horizontal wind components correspond to a summation of sinusoidal function with random parameters (magnitude, frequency and phase),

$v_{w_{i}(t)}=v_{w_{i}}^{s}+\sum_{k=1}^{n} \sqrt{\Delta_{\omega_{k}} \Phi_{k}} \sin \left(\omega_{n_{k}} t+\epsilon_{k}\right)$ with $i:\{x, z\}$

where $v_{w}^{s} \in \mathbb{R}$ is the static wind, $\omega_{n} \in \mathbb{R}$ is the natural frequency, $\Delta_{\omega} \in \mathbb{R}$ is frequency samples, $\epsilon \in \mathbb{R}$ is a random phase value and

$$
\begin{aligned}
\Phi_{x} & =\sigma_{x}^{2} \frac{2 L_{x}}{\pi} \frac{1}{1+\left(L_{x} \omega_{n}\right)^{2}} \\
\Phi_{z} & =\sigma_{z}^{2} \frac{2 L_{x}}{\pi} \frac{1+3\left(L_{x} \omega_{n}\right)^{2}}{\left(1+\left(L_{x} \omega_{n}\right)^{2}\right)^{2}}
\end{aligned}
$$

which include the turbulence intensities in the $x$ and $z$ directions, i. e. $\sigma_{x}, \sigma_{z} \in \mathbb{R}$ respectively, $L_{x} \in \mathbb{R}$ and $L_{z} \in \mathbb{R}$ are the turbulence scale lengths in the $x$ and $z$ directions. In low altitude regions $(z<1000 \mathrm{ft}(304.8 \mathrm{~m}))$, the turbulence length and intensities can be obtained according to [8], by:

$$
\begin{aligned}
L_{z} & =|z| \\
L_{x} & =\frac{z}{(0.177+0.000823 z)^{1.2}} \\
\sigma_{z} & =0.1 W_{20} \\
\sigma_{x} & =\frac{\sigma_{z}}{(0.177+0.000823 z)^{0.4}}
\end{aligned}
$$

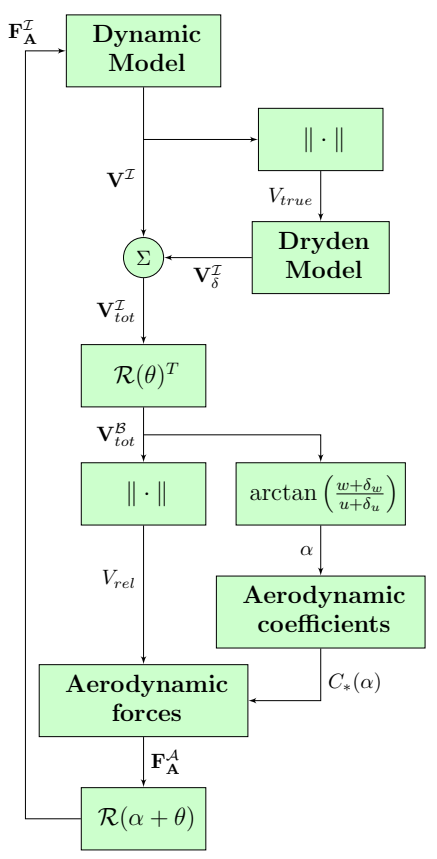

Fig. 2. MAV's aerodynamics modeling process

where $W_{20}$ is the wind speed at $20 \mathrm{ft}(6 \mathrm{~m})$ above ground and it is defined according to the turbulence level. For this instance, let us consider moderate turbulence level so $W_{20}=$ $15.4333 \mathrm{~m} / \mathrm{s}$.

The aerodynamic frame is shaped via the velocities in the body frame. Then it follows that the AoA is given as

$$
\alpha=\tan ^{-1}\left(\frac{w}{u}\right)
$$

and

$$
V_{\text {rel }}=\sqrt{u^{2}+w^{2}}
$$

The velocity in the body frame are found to depend on inertial terms, i. e.

$$
\begin{aligned}
u & =\dot{x} \cos \theta-\dot{z} \sin \theta \\
w & =\dot{x} \sin \theta+\dot{z} \cos \theta
\end{aligned}
$$

Considering the late, let us update (Eq. 4) and (Eq. 5) including (Eq. 1) with the wind disturbances, so they can be rewritten as

$$
\begin{aligned}
u & =\left(\dot{x}+v_{w_{x}}\right) \cos \theta-\left(\dot{z}+v_{w_{z}}\right) \sin \theta \\
w & =\left(\dot{x}+v_{w_{x}}\right) \sin \theta+\left(\dot{z}+v_{w_{z}}\right) \cos \theta
\end{aligned}
$$

Now we proceed to present the expressions for the aerodynamic forces exerted on the rotorcraft's aerodynamic center (AC):

$$
\begin{aligned}
D & =\frac{1}{2} V_{\text {rel }}^{2} \rho S C_{D} \\
L & =\frac{1}{2} V_{\text {rel }}^{2} \rho S C_{L}
\end{aligned}
$$

The aerodynamic forces vector includes $D$ and $L, \mathbf{F}_{A}^{A}=$ $(-D, L) \in \mathbb{R}^{2}$ whose projection into the inertial frame (from the aerodynamic frame $\mathcal{A}$ ) is written, in its scalar version, as:

$$
\begin{aligned}
& \delta_{a_{x}}=L \sin (\theta+\alpha)-D \cos (\theta+\alpha) \\
& \delta_{a_{z}}=L \cos (\theta+\alpha)+D \sin (\theta+\alpha)
\end{aligned}
$$


Since the geometry of the airframe and the cargo are assumed the same, the aerodynamic forces are proportional. However, the cargo forces disturb the rotorcraft by a couple, leading to

$$
\begin{aligned}
& \delta_{u_{c}}=L \sin (\alpha)-D \cos (\alpha) \\
& \delta_{v_{c}}=L \cos (\alpha)+D \sin (\alpha)
\end{aligned}
$$

obtaining the aerodynamic-based torque (see Fig. 1) including the rotation motion $\gamma$,

$$
\tau_{\delta_{a}}=-\delta_{u_{c}} l_{p} \sin \gamma+\delta_{v_{c}} l_{p} \cos \gamma
$$

\section{Dynamic Equations of Motion}

The equations of motion describing the overall motion behavior of the interactive aerial robot are written and given as (for details see [14] and [15]):

$$
\begin{aligned}
u_{x}= & (M+m) \ddot{x}-m l \ddot{\gamma} \cos \gamma+m l \dot{\gamma}^{2} \sin \gamma \\
u_{z}= & (M+m) \ddot{z}+m l \ddot{\gamma} \sin \gamma+m l \dot{\gamma}^{2} \cos \gamma+ \\
& (M+m) g
\end{aligned}
$$

Recalling that for the translational equation, displacement along the $x$-axis is underactuated via the pitch dynamics [16], then $u_{x}=T \sin \theta \in \mathbb{R}$ and $u_{z}=T \cos \theta \in \mathbb{R}$ correspond to the thrust vector projection due to the pitch angle. The equations describing the rotational dynamics of the multi-body system are defined as:

$$
\begin{aligned}
u_{\theta}= & I_{r} \ddot{\theta} \\
u_{\gamma}= & m l^{2} \ddot{\gamma}-m l \ddot{x} \cos \gamma+\ddot{z} m l \sin \gamma+ \\
& m g l \sin \gamma
\end{aligned}
$$

where $u_{\theta}=\tau_{\theta} \in \mathbb{R}$ and $u_{\gamma}=\tau_{\gamma} \in \mathbb{R}$ stand for the torque applied by the rotors' differential thrust and for the robotic arm, respectively. However, provided that both torques are exerted about the same axis $\left(e_{2}\right)$, thus, the robotic arm motion becomes a twofold disturbance affecting with torque actuators and with center of gravity shifting. Thus (Eq.16) becomes

$$
u_{\theta}+m l^{2} \ddot{\gamma}=I_{r} \ddot{\theta}
$$

Let us rewrite this 3DOF model by splitting the nominal model and a lumped disturbance term containing the dynamic couplings as well as the aerodynamic-due forces and torques, for the translational and rotational dynamics.

$$
\begin{aligned}
\ddot{x} & =\frac{1}{M}(T \sin \theta)+\Delta_{x}(t) \\
\ddot{z} & =\frac{1}{M}(T \cos \theta-M g)+\Delta_{z}(t) \\
\ddot{\theta} & =\frac{1}{I_{r}} \tau_{\theta}+\Delta_{\theta}(t) \\
\ddot{\gamma} & =\frac{1}{m l^{2}} \tau_{\gamma}+\Delta_{\gamma}(t)
\end{aligned}
$$

where $\Delta_{x}(t), \Delta_{z}(t)$ and $\Delta_{\theta}(t) \in \mathbb{R}$ comprise the parametric, dynamic and aerodynamic uncertainties, i.e.

$$
\begin{aligned}
\Delta_{x}(t)= & \frac{1}{M}\left(u_{x}+m l \ddot{\gamma} \cos \gamma-m l \dot{\gamma}^{2} \sin \gamma+\ldots\right. \\
& -m \ddot{x})+\delta_{a_{x}} \\
\Delta_{z}(t)= & \frac{1}{M}\left(u_{z}-M g-m l \ddot{\gamma} \sin \gamma+\right. \\
& \quad \ldots-m g-m \ddot{z})+\delta_{a_{z}} \\
\Delta_{\theta}(t)= & \frac{1}{I_{r}}\left(u_{\gamma}+m l \ddot{x} \cos \gamma-\ddot{z} m l \sin \gamma+\ldots\right. \\
& -m g l \sin \gamma)+\tau_{\delta_{a}} \\
\Delta_{\gamma}(t)= & \frac{1}{m l^{2}}\left(u_{\gamma}+m l \ddot{x} \cos \gamma-\ddot{z} m l \sin \gamma+\ldots\right. \\
& -m g l \sin \gamma)+\tau_{\delta_{a}}
\end{aligned}
$$

\section{CONTROL}

Let us consider the classical terms for the desired thrust and attitude,

$$
T^{d}=\left\|\left(u_{x}, u_{z}\right)^{T}\right\| \text { and } \theta^{d}=\tan ^{-1}\left(\frac{u_{x}}{u_{z}+M g}\right)
$$

whose insertion in (Eq. 23) and (Eq. 24) defines a linearization loop of the nominal system resulting in the following expressions

$$
\begin{aligned}
\ddot{x} & =\frac{1}{M}\left(u_{x}\right)+\Delta_{x}(t) \\
\ddot{z} & =\frac{1}{M}\left(u_{z}\right)+\Delta_{z}(t)
\end{aligned}
$$

It is noteworthy to mention that the disturbance remain. Let us regroup (Eq 21), (Eq 22), (Eq 28) and (Eq 29) as follows

$$
\ddot{\chi}_{i}=\frac{1}{k_{i}} \nu_{i}+\Delta_{i}(t) \text { with } i \in\{x, y, \theta, \gamma\}
$$

with $k_{x}=M, k_{z}=M, k_{\theta}=I_{r}$ and $k_{x}=m l^{2}$. Likewise, $\nu_{x}=u_{x}, \nu_{z}=u_{z}, \nu_{\theta}=\tau_{\theta}$ and $\nu_{\gamma}=\tau_{\gamma}$.

Now, for trajectory tracking purposes, $\chi_{i} \equiv \chi_{i}^{d}$, let us define a sliding surface $s=0[17][18]$,

$$
s_{i}=\dot{\varepsilon}_{i}+\lambda \varepsilon_{i}+\xi
$$

with $\varepsilon_{i}=\chi_{i}-\chi_{i}^{d}$ and $\xi$ an integral term of the errors. The time-derivative of (Eq. 31)

$$
\dot{s}_{i}=\ddot{\varepsilon}_{i}+\lambda_{i} \dot{\varepsilon}_{i}=\frac{1}{k_{i}} \nu_{i}+\Delta_{i}(t)-\ddot{\chi}_{i}^{d}+\lambda \dot{\varepsilon}_{i}
$$

Thus, the controller that attempts to achieve $\dot{s}=0$ ( i.e. to stay on the sliding surface) is given as

$$
\nu_{i}=-k_{p} \chi-k_{d} \chi-k_{s} \tanh (s)+\ddot{\chi}_{i}^{d}-\lambda_{i} \dot{\varepsilon}_{i}-\xi
$$

\section{NumericAl Simulations}

In this section, we present a detailed simulation study showing the results of the actual proposed strategy regarding rapid in-flight grasping, transporting and placing a payload within realistic aerodynamic conditions. 

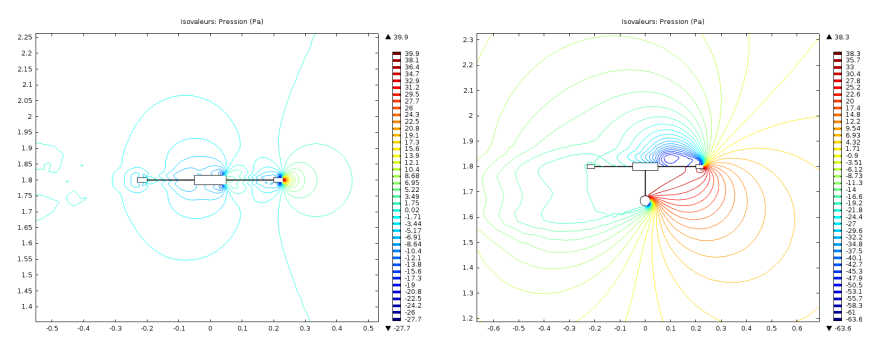

Fig. 3. Pressure schemes: (left) without cargo and (left) with cargo
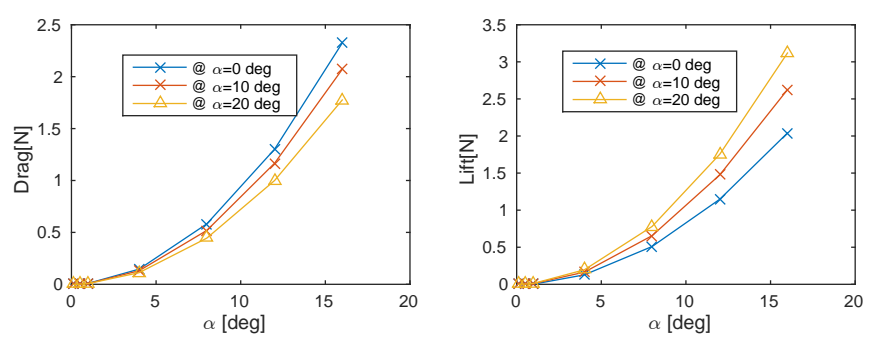

Fig. 4. Aerodynamic forces: (left) lift and (left) drag

\section{A. Aerodynamic simulations}

In order to computed the aerodynamic parameters of the flying robot presented in previous sections, we have used StarCCM+. This section describes the overall aerodynamic behavior showing the air slipstream's pressure exerted on the aerial robot in order to determine the forces in function of velocity and AoA. We have considered a bi-dimensional scenario where the MAV is studied at different velocities and positions for comparison purposes. Moreover, this will provide specific information about the least-drag configuration during interactive operation.

Fig. 3 shows the pressure contour plot obtained for different angles for both loaded and unloaded operations meanwhile, Fig. 4 shows the aerodynamic forces acting on the system.

\section{B. Trajectory Generator}

The commanded trajectory for the altitude is based on a co-sinusoidal function whose parameters (frequency and amplitude) are updated based on the object location $\left(x_{o b j}, z_{o b j}\right)$ in order to reach the grasping and deploying target points. The reference trajectory for the flight path along $x$-axis aims at defining a specific speed pattern to reach the aforementioned target points. Such time-parameterized reference trajectory is configured as follows

$$
\begin{gathered}
z^{r e f}(t)=A_{z}+\left[A_{z}-\left(l+z_{o b j}\right)\right] \cos \left(\frac{\pi}{T_{o p}} t\right) \\
x^{r e f}(t)=v_{h} t
\end{gathered}
$$

where $v_{h} \in \mathbb{R}$ is the desired horizontal velocity, $T_{o p} \in \mathbb{R}$ is the punctual action time, either the grasping time $T_{o p}$ : $T_{\text {grasp }}$ or the deploying time, $T_{o p}: T_{\text {deploy }}$, with

$$
T_{o p}=\frac{v_{h}}{x_{o b j}},
$$

and $A_{z} \in \mathbb{R}$ represents the altitude of the vehicle during the interactive operation, whose value might be updated in function of the operation.

\section{Simulation Results}

In order to evaluate the performance of the proposed control law via simulation, the parameters of the quad-rotor pendulum system considered for the analysis were set to be the ones presented next in Table I.

TABLE I

QUAD-ROTOR PENDULUM SYSTEM PARAMETERS

\begin{tabular}{cl}
\hline Parameter & Value \\
\hline$M, m, I_{r}, \ell$ & $0.5 \mathrm{~kg}, 0.25 \mathrm{~kg}, 0.1250 \mathrm{~kg} \mathrm{~m} \mathrm{~m}^{2}, 0.5 \mathrm{~m}$ \\
\hline
\end{tabular}

The initial position of the object to be deployed was established at $\left(x_{o}, z_{o}\right)=(10,2)[\mathrm{m}]$.

Fig. 5 and Fig. 7 depict the performance of the proposed path/control strategy to deal with a rapid in-flight retrieving task. Specifically, Fig. 5 shows that the trajectory tracking objective is fulfilled for both stages, pre-retrieving $(t<$ $1.428 \mathrm{sec}$ ) and specially for post-retrieving $(t \geq 1.428 \mathrm{sec})$ where a disturbing torque arises from the payload drag. Fig. 6 exhibits that the attitude is successfully tracking the control reference provided by the outer-loop translational dynamics, while the robotic arm is commanded to remain in vertical position. The aerodynamic forces and manipulator's torque (shown in Fig. 7) increase during the post-retrieving stage as a result of the drag and lift exerted on the payload. In this figure, it is also depicted the wind velocity. Lastly, Fig. 8 shows the two dimensional motion of the rotorcraft during the retrieving task. It is worthy to mention that keeping the robotic arm in vertical position is twofold, on one hand, it remains aligned with the rotorcraft's center of gravity reducing torques disturbances due to the gripper, but on the other hand, this position corresponds to the maximal aerodynamic-based torque provided by the robotic arm.

\section{CONCLUding REMARKS}

In the actual paper, we have addressed the problem of aerial grasping within windy conditions. We have presented in detail the planar (3DOF) dynamics and aerodynamics associated to a rotorcraft endowed with a $1 \mathrm{DOF}$ robotic arm. The Dryden wind model was used to complete such dynamics. The ISC is used to stabilize the overall system, where the dynamic and the aerodynamic-based couplings are regrouped into a lumped disturbance term. In this regard, a tunable co-sinusoidal pattern is proposed to reach the target, whose parameters can be defined, for a static object, or dynamically updated for the case of a mobile target. A detailed simulation stage was conducted considering a realistic scenario, i.e. including the actual aerodynamic parameters of the interactive rotorcraft as well as the wind model. The current study suggests that the next stage is to identify operational regimes and/or patterns combinations regarding to minimize adverse aerodynamics while effective grasping and transportation are guaranteed. 


\section{REFERENCES}
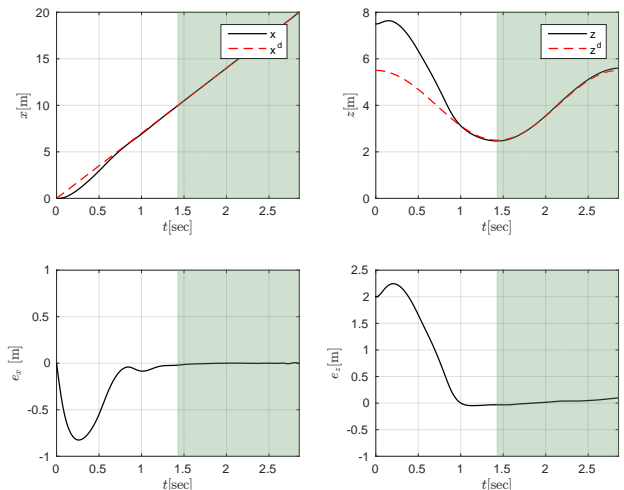

Fig. 5. Trajectory performance: translational states evolution
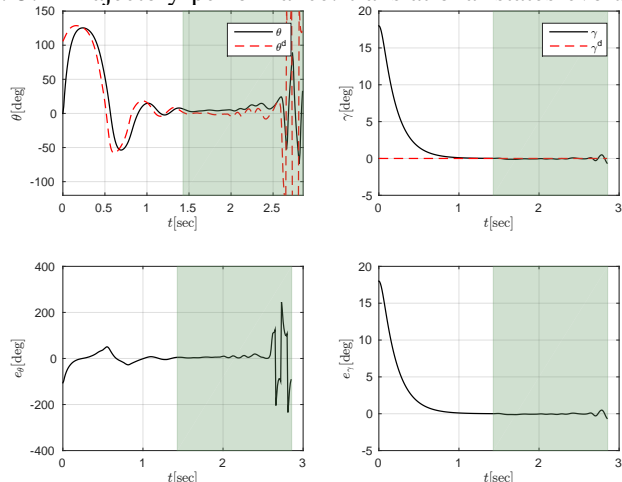

Fig. 6. Trajectory performance: rotational states evolution
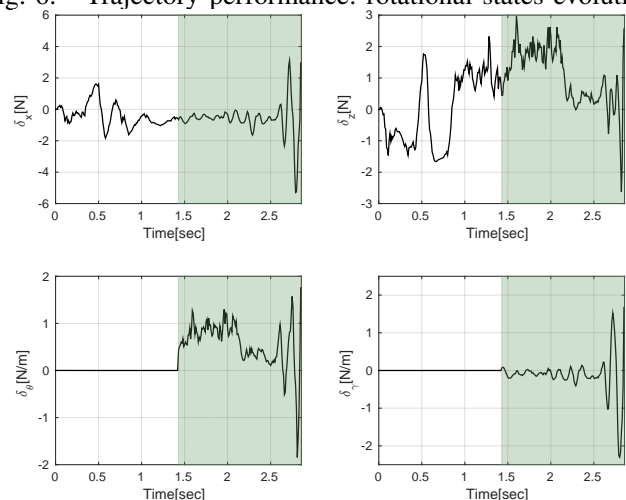

Fig. 7. Total disturbances
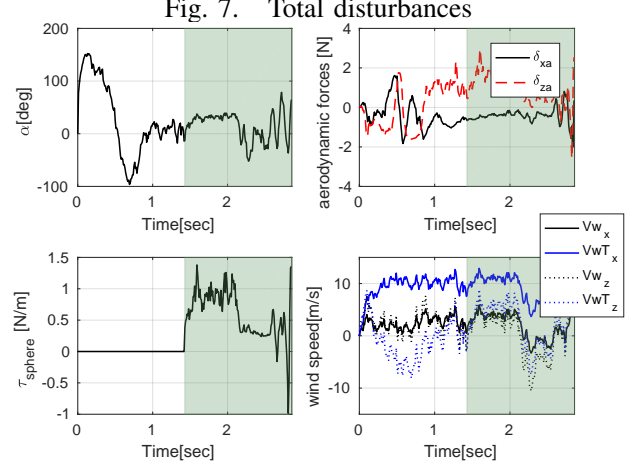

Fig. 8. Aerodynamic behavior
[1] D. R. Nelson, D. B. Barber, T. W. McLain, and R. W. Beard, "Vector field path following for small unmanned air vehicles," in 2006 American Control Conference. IEEE, 2006, pp. 7-pp.

[2] T. G. McGee and J. K. Hedrick, "Path planning and control for multiple point surveillance by an unmanned aircraft in wind," in 2006 American Control Conference. IEEE, 2006, pp. 6-pp.

[3] A. Ratnoo, P. Sujit, and M. Kothari, "Adaptive optimal path following for high wind flights," IFAC Proceedings Volumes, vol. 44, no. 1, pp. 12985-12990, 2011.

[4] A. Brezoescu, P. Castillo Garcia, and R. Lozano, "Straight-line path following in windy conditions," in Proceedings of the International Conference on Unmanned Aerial Vehicle in Geomatics $(U A V-g)$, Zurich, Suisse, Sep 2011, pp. -. [Online]. Available: http://hal.archives-ouvertes.fr/hal-00660384

[5] S. L. Waslander and C. Wang, "Wind disturbance estimation and rejection for quadrotor position control," in AIAA Infotech Aerospace Conference and AIAA Unmanned... Unlimited Conference, Seattle, WA, 2009.

[6] J. Colorado, A. Barrientos, A. Martinez, B. Lafaverges, and J. Valente, "Mini-quadrotor attitude control based on hybrid backstepping \& frenet-serret theory," in Robotics and Automation (ICRA), 2010 IEEE International Conference on. IEEE, 2010, pp. 1617-1622.

[7] C. Hancer, K. Oner, E. Sirimoglu, E. Cetinsoy, and M. Unel, "Straightline path following in windy conditions," in 49th IEEE Conference on Decision and Control (CDC), 2010.

[8] B. Ahmed and F. Kendoul, "Flight control of a small helicopter in unknown wind conditions," in 49th IEEE Conference on Decision and Control (CDC). IEEE, 2010, pp. 3536-3541.

[9] D. Mellinger, M. Shomin, and V. Kumar, "Control of quadrotors for robust perching and landing," in Proceedings of the International Powered Lift Conference, 2010, pp. 205-225.

[10] N. R. Kuntz and P. Y. Oh, "Towards autonomous cargo deployment and retrieval by an unmanned aerial vehicle using visual servoing," in ASME 2008 International Design Engineering Technical Conferences and Computers and Information in Engineering Conference. American Society of Mechanical Engineers, 2008, pp. 841-849.

[11] P. E. Pounds, D. Bersak, and A. Dollar, "Grasping from the air: Hovering capture and load stability," in Robotics and Automation (ICRA), 2011 IEEE International Conference on, May 2011, pp. 24912498.

[12] J. Thomas, G. Loianno, K. Sreenath, and V. Kumar, "Toward Image Based Visual Servoing for Aerial Grasping and Perching," in ICRA. IEEE, 2014.

[13] G. J. Ghadiok, V. and W. Ren, "Autonomous indoor aerial gripping using a quadrotor," in In 2011 IEEE/RSJ International Conference on Intelligent Robots and Systems, 2012.

[14] J. Escareno, A. Belbachir, T. Raharijaona, and S. Bouchafa, "Generalized disturbance estimation via eslkf for the motion control of rotorcraft having a rod-suspended load," in 13th International Conference on Informatics in Control, Automation and Robotics (ICINCO),, 2016, pp. 526-533.

[15] J. Escareno, M. Rakotondrabe, G. Flores, and R. Lozano, "Rotorcraft mav having an onboard manipulator: Longitudinal modeling and robust control," in European Control Conference (ECC), 2013, July 2013, pp. 3258-3263.

[16] M. Fumagalli, R. Naldi, A. Macchelli, R. Carloni, S. Stramigioli, and L. Marconi, "Modeling and control of a flying robot for contact inspection," pp. 3532-3537, Oct 2012.

[17] J.-J. E. Slotine and W. Li, Applied nonlinear control. Englewood Cliffs (N.J.): Prentice Hall, 1991. [Online]. Available: http: //opac.inria.fr/record=b1132812

[18] S. L. Waslander, G. M. Hoffmann, J. S. Jang, and C. J. Tomlin, "Multiagent quadrotor testbed control design: integral sliding mode vs. reinforcement learning," in 2005 IEEE/RSJ International Conference on Intelligent Robots and Systems, Aug 2005, pp. 3712-3717. 